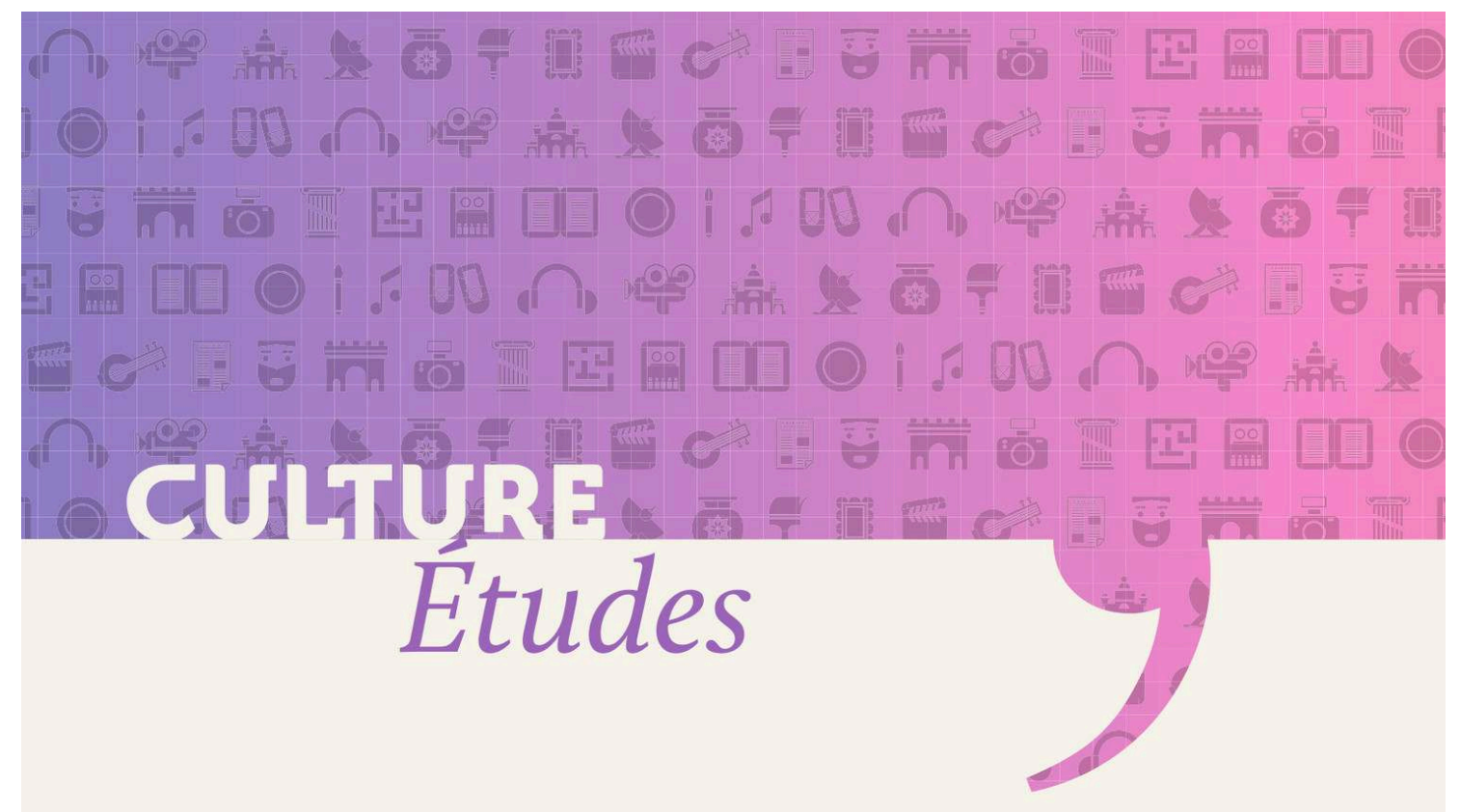

\title{
La lente féminisation des professions culturelles
}

\author{
Marie Gouyon \\ Frédérique Patureau \\ Gwendoline Volat
}
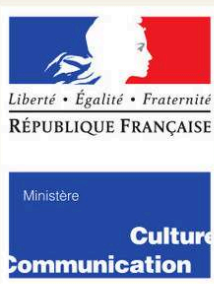

2016-2

Ministère de la Culture et de la Communication Département des études, de la prospective et des statistiques 


\section{La lente féminisation des professions culturelles}

The Gradual Feminisation of the Cultural Professions

Marie Guyon, Frédérique Patureau et Gwendoline Volat

Éditeur : Département des études, de la prospective et des statistiques

Lieu d'édition : Paris

Année d'édition : 2016

Date de mise en ligne : 23 février 2018

Collection : Culture études

ISBN électronique : 9782111399327

\section{Sobooks}

http://books.openedition.org

Édition imprimée

Nombre de pages : 22

Référence électronique

GUYON, Marie ; PATUREAU, Frédérique ; et VOLAT, Gwendoline. La lente féminisation des professions culturelles. Nouvelle édition [en ligne]. Paris : Département des études, de la prospective et des statistiques, 2016 (généré le 25 avril 2021). Disponible sur Internet : <http://books.openedition.org/ deps/1203>. ISBN : 9782111399327.

(C) Département des études, de la prospective et des statistiques, 2016

Creative Commons - Attribution - Pas d'Utilisation Commerciale 3.0 non transposé - CC BY-NC 3.0 


\section{CUMURF Etudes}

\section{La lente féminisation des professions culturelles}

Marie Gouyon

Frédérique Patureau

Gwendoline Volat

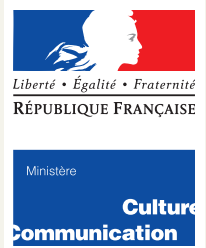

2016-2

Ministère de la Culture et de la Communication

Département des études,

de la prospective

et des statistiques 


\title{
La lente féminisation des professions culturelles
}

\author{
Marie Gouyon*, \\ Frédérique Patureau*, \\ Gwendoline Volat*
}

Depuis les années 1960, la croissance du taux d'activité féminine est l'une des transformations majeures du monde du travail, et les professions culturelles n'y font pas exception : la part des femmes au sein de ces professions est ainsi passée de $39 \%$ à $43 \%$ de 1991 à 2013. Presque tous les métiers fortement masculins au début des années 1990 comme les métiers d'art, les architectes et les photographes, par exemple, se sont ouverts aux femmes. Pour autant, la part des femmes demeure inférieure à la moyenne nationale, où l'activité féminine atteint $48 \%$ de l'ensemble des professions, un constat qui peut surprendre au regard de la surreprésentation des femmes en termes de participation culturelle.

En se développant, l'emploi féminin a largement épousé les spécificités de l'emploi propres aux professions culturelles, notamment leur plus grande flexibilité: les professionnelles de la culture sont, plus souvent que l'ensemble des actives, non salariées ou salariées sur contrats à durée limitée. Elles travaillent plus souvent à temps partiel, avec des horaires variables et atypiques, en soirée et le week-end.

Surtout, leur accès aux professions artistiques et culturelles conforte la règle de la surqualification des femmes en termes de niveau de diplôme : en 2013, plus d'une active de ces professions sur deux est titulaire d'un diplôme égal ou supérieur à bac +3 , contre $40 \%$ de leurs homologues masculins - une surqualification d'autant plus forte qu'il s'agit de métiers traditionnellement exercés par des hommes.

* Chargées d'études au Département des études, de la prospective et des statistiques, ministère de la Culture et de la Communication. 
Depuis les années 1960, la croissance du taux d'activité féminine constitue l'une des transformations majeures du monde du travail. Cette progression ininterrompue et de grande ampleur conduit aujourd'hui, pour la première fois, à une répartition quasiment paritaire des actifs ( $48 \%$ de femmes actives en emploi en 2013 contre $37 \%$ seulement en $1975^{1}$ ).

Les professions culturelles ${ }^{2}$ ne sont pas restées à l'écart de cette tendance de fond, comme le montre l'analyse rétrospective des résultats de l'enquête Emploi (Insee) sur plus de vingt ans, entre 1991 et 2013 ("Sources et méthodes», p. 19) : la part des femmes y progresse de façon régulière et dans une proportion tout à fait comparable à celle observée dans l'ensemble de la population active (graphique 1).

\section{Graphique 1 - Évolution de la part des femmes dans l'ensemble des professions culturelles et des actifs en emploi, 1991-2013}

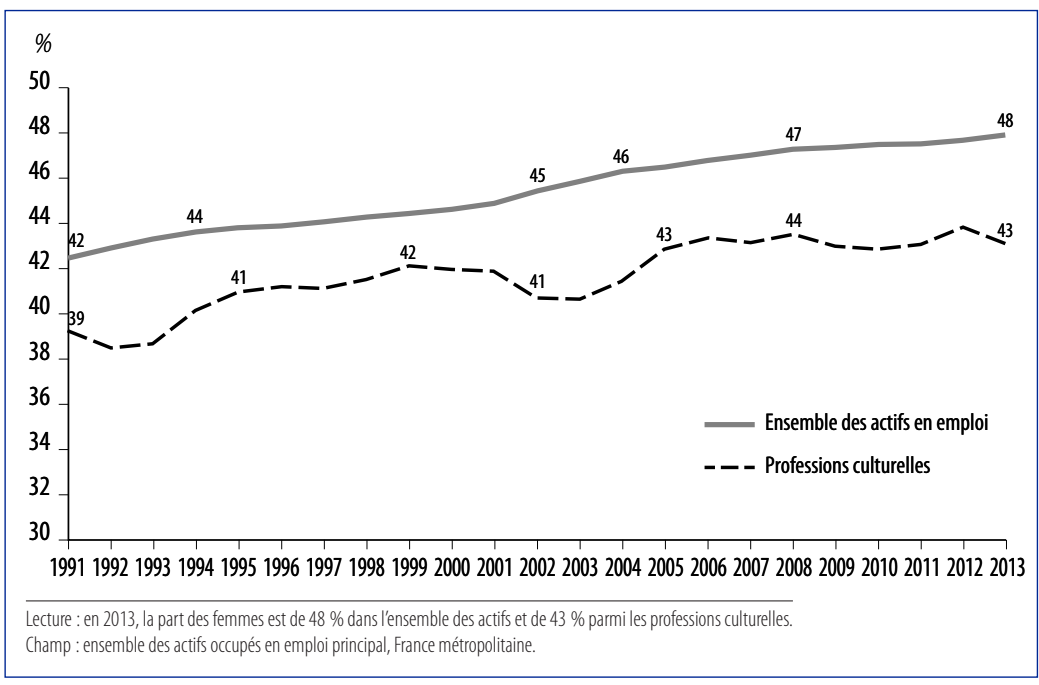

Source : Enquêtes Emploi, Insee/DEPS, Ministère de la Culture et de la Communication, 2016

Pourtant, cette part demeure, comme il y a vingt ans, inférieure à la moyenne nationale : $39 \%$ seulement de femmes dans les métiers culturels en 1991 et $43 \%$ en 2013, contre respectivement $43 \%$ et $48 \%$ dans l'ensemble de la population active. Un constat dont la permanence interpelle, s'agissant d'un champ d'activité volontiers considéré comme plus ouvert, plus affranchi des contraintes et des déterminations sociales ${ }^{3}$.

1. Source : Insee, enquêtes Emploi.

2. Le terme "profession » est employé ici de façon générique, essentiellement par référence à la nomenclature des "Professions et catégories socioprofessionnelles» (PCS) qui sert de cadre à la présente analyse et par souci de simplification stylistique. II ne renvoie donc pas aux seules activités professionnelles formellement encadrées et réglementées (la profession d'architecte ou celle de conservateur, par exemple), plutôt minoritaires dans le champ culturel, mais à l'ensemble particulièrement hétérogène des activités professionnelles (au sens anglo-saxon d'occupations) relevant du champ d'intervention du ministère de la Culture et de la Communication (voir Les disparités de revenus dans les professions culturelles, p. 18). II sera employé ici indifféremment au terme «métier » - lui aussi utilisé dans une acception très générique, ne renvoyant donc pas exclusivement au cœur de compétence, de qualification et de savoir-faire spécifique attaché à telle ou telle activité professionnelle.

3. Marie BusCATTO, "L'art sous l'angle du genre ou révéler la normativité des mondes de l'art », dans Alain Quemin et Glaucia Villas Boas (sous la dir. de), Art et société, Marseille, OpenEdition Press, 2016. 


\section{Un constat en forme de paradoxe}

Il prend même l'allure d'un paradoxe lorsqu'on le rapproche d'autres observations attestant, exactement à l'inverse, d'une implication privilégiée des femmes dans le champ artistique et culturel. Toutes les enquêtes sur la fréquentation des équipements culturels, sur les pratiques artistiques et culturelles en amateur font en effet le constat, à intervalles réguliers, d'une surreprésentation féminine qui tend à s'amplifier notablement et continûment sur période longue, en France comme ailleurs 4 .

Il en va de même lorsqu'on observe la composition des populations étudiantes qui fréquentent les très nombreuses formations artistiques et culturelles à visée professionnelle : déjà majoritaire au début des années 1990, la part des femmes y est, depuis cette date, en développement continu ${ }^{5}$.

La sphère des activités rémunérées serait-elle l'exception qui confirme la règle d'un univers massivement investi par les femmes ?

La permanence de cette relative sous-représentation des femmes dans l'exercice professionnel d'une activité culturelle interroge d'autant plus que le nombre total d'actifs au sein de ces métiers a précisément connu, au cours des vingt dernières années, une forte expansion, supérieure à celle observée dans l'ensemble de la population active ${ }^{6}$. II faut donc en conclure que cet essor quantitatif exceptionnel n'a pas permis aux femmes d'y investir une place au moins comparable à celle qu'elles occupent désormais dans l'ensemble de la population active.

Comprendre les raisons de ce décalage des métiers culturels par rapport au niveau atteint par la féminisation de la population active relève à l'évidence d'une série d'analyses approfondies et sur période longue, tant le " genre » d'un métier et ses variations dans le temps doivent d'abord aux conditions sociohistoriques de l'émergence de ce métier, à ses caractéristiques propres (technologiques, réglementaires...) et à l'évolution de celles-ci. En outre, la très grande diversité des métiers artistiques et culturels du point de vue de l'emploi (emplois salariés et non salariés; emplois de création et d'interprétation ; emplois artisanaux, industriels, commerciaux; emplois à l'accès réglementé ou à l'inverse, largement ouverts, etc.) impose aussi, plus que dans tout autre champ peut-être, des approfondissements métier par métier, ou par familles de métiers. De nombreux travaux de recherche existent déjà ou sont en cours ${ }^{7}$ et c'est de la capitalisation de leurs résultats que des interprétations peuvent être formulées.

Il s'agit ici de prendre la mesure statistique de la progression du mouvement de féminisation au sein d'un ensemble défini de professions culturelles sur vingt ans et d'en dresser les principales caractéristiques.

4. Olivier Donnat, la Féminisation des pratiques culturelles, Paris, Ministère de la Culture et de la Communication, DEPS, coll. "Développement culturel », n 147, juin 2005; Angèle CHRISTIN et Olivier Donnat, Pratiques culturelles en France et aux États-Unis. Éléments de comparaison 1981-2008, Paris, Ministère de la Culture et de la Communication, DEPS, coll. "Culture études», n 2014-1, 2014 et Sylvie OCTOBRE, la Fabrication sexuée des goûts culturels, Paris, Ministère de la Culture et de la Communication, DEPS, coll. « Développement culturel », n 150, décembre 2005.

5. Entre 1990-1991 et 2011-2012, la part des étudiantes dans les établissements d'enseignement artistique et culturel (écoles d'architecture, écoles supérieures artistiques et culturelles) est passée ainsi de $50 \%$ à $59 \%$ (source : Ministère de l'Enseignement supérieur et de la Recherche, DGESIP-DGRI SIES).

6. Marie Gouyon et Frédérique Patureau, Vingt ans d'évolution de l'emploi dans les professions culturelles, Paris, Ministère de la Culture et de la Communication, DEPS, coll. « Culture chiffres », n 2014-6, 2014.

7. Ces travaux sont nombreux et explorent sous I'angle du genre différents métiers du champ culturel (on peut citer à titre d'exemples les recherches de Delphine NAUDIER sur les femmes écrivains, de Marie Buscatto, Philippe Coulangeon et Hyacinthe Ravet sur les mondes de la musique, de Janine Rannou et Ionela ROHARIK sur celui de la danse, ou encore de Nathalie LAPEYRE sur les architectes). On se reportera tout particulièrement aux recherches fédérées depuis le milieu des années 1990 par le groupe de recherche "Marché du travail et genre en Europe » (MAGE - CNRS) qui ont donné lieu à des publications, colloques et journées d'études spécialement consacrés aux mondes de l'art et de la culture (Marlaine CACOUAULT-BITAUD et Hyacinthe RAVET [sous la dir. de], numéro spécial « Les femmes, les arts et la culture », Travail, genre et sociétés, n 19, 2008; Marie BusCATTO, Catherine MARRY et Delphine NAUDIER [sous la dir. de], Actes des journées d'études Travail, genre et art, 2008). 
Fondée sur la seule exploitation d'une série chronologique des enquêtes Emploi de l'Insee, l'analyse est contrainte par les variables de cette enquête et limitée à cellesci. Certains éléments dont l'importance est attestée par toutes les analyses de l'emploi et des professions selon le genre sont ainsi absents de l'analyse: les disparités de revenu (voir "Les disparités de revenus dans les professions culturelles », p. 18), ou celles des positions hiérarchiques occupées dans les différents métiers, par exemple, ne peuvent être appréhendées avec un tel outil, car ces variables sont absentes de l'enquête Emploi ou non exploitables de manière fiable. De même, la succession annuelle des enquêtes Emploi produit une série de photographies de la part des femmes dans les différents métiers, juxtaposées d'une année à l'autre, mais ne dit rien des trajectoires professionnelles des femmes et des hommes, c'est-à-dire de la capacité des unes et des autres à s'insérer, durablement ou non, au sein de ces métiers - autre élément de différenciation important dans l'analyse comparée des situations professionnelles des hommes et des femmes. L'intérêt de cette source, commune à l'ensemble des professions considérées et stable dans le temps, réside donc avant tout dans la mesure des évolutions et dans la comparaison interne, d'une profession (ou groupe de professions) à l'autre.

On s'attachera, dans un premier temps, à une quantification du mouvement de féminisation : celui-ci a concerné tous les métiers, mais dans des proportions et avec des caractéristiques associées parfois très différentes d'une profession à l'autre qu'il convient donc de souligner. Dans un deuxième temps, ce sont les spécificités de l'emploi féminin qui seront examinées, notamment pour savoir si l'on retrouve, dans ce champ particulier, des caractéristiques identiques à celles observées dans l'ensemble de la population active : la surreprésentation du salariat et du travail à temps partiel dans l'emploi féminin, par exemple. On reviendra enfin, à partir des différences observées par profession, sur les traits communs qui peuvent apparaître, sur la durée, comme ayant favorisé le mouvement de féminisation.

\section{Un mouvement de fond qui a touché toutes les professions}

En vingt ans, la physionomie du champ professionnel constitué par les métiers artistiques et culturels sous l'angle de la répartition par sexe s'est profondément modifiée.

En 1991, dans un paysage d'ensemble au sein duquel les femmes ne représentaient qu'un gros tiers des effectifs totaux, l'observation de premier abord était celle de métiers très fortement sexués : sept groupes de professions sur treize apparaissaient comme nettement masculins et comptaient au moins deux tiers d'hommes, et deux sur treize se révélaient, à l'inverse, largement féminins (au moins deux tiers de femmes). Seuls deux groupes de métiers présentaient, au début des années 1990, une répartition de leurs actifs assez proche de la parité : les professionnels des arts graphiques, de la mode et de la décoration (graphistes, stylistes, designers...) d'une part, les auteurs littéraires, d'autre part (graphique 2).

Cette faible mixité au sein des différentes professions n'était pas propre au champ culturel : on l'observait également au niveau de l'ensemble de la population active et le mouvement d'afflux massif des femmes sur le marché du travail au cours des dernières décennies n'a pas altéré notablement ce phénomène ${ }^{8}$. 


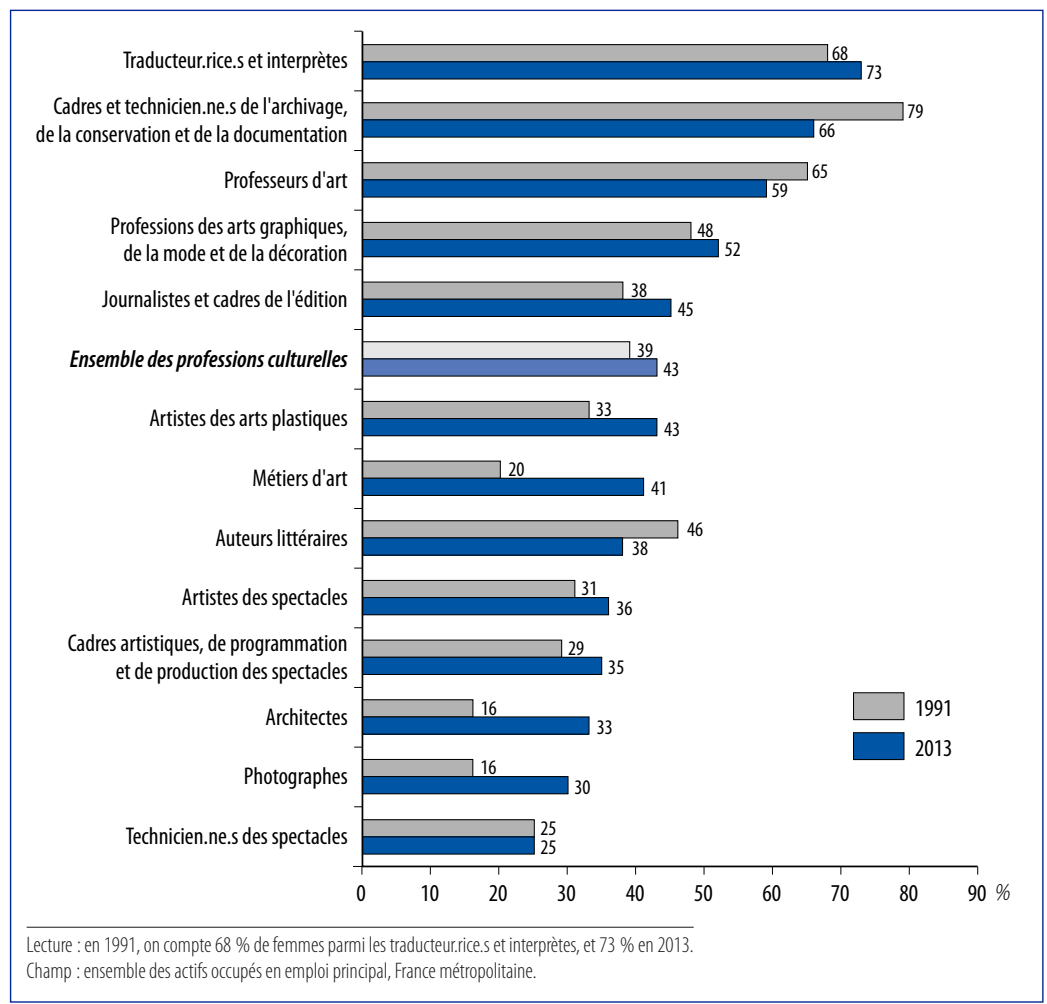

Source : Enquêtes Emploi, Insee/DEPs, Ministère de la Culture et de la Communication, 2016

En 2013, le changement mesuré est important : cinq groupes de professions culturelles sur treize présentent désormais une répartition sexuée sensiblement équilibrée de leurs actifs (graphique 2), parmi lesquels le métier d'artiste plasticien, très fortement masculin en 1991 (67\% d'hommes) et celui de professeur d'art, très féminin au contraire ( $65 \%$ de femmes).

Plus généralement, tous les métiers fortement masculins vingt ans plus tôt se sont ouverts aux femmes - et certains d'entre eux, de façon spectaculaire : c'est le cas tout particulièrement des métiers d'art et du métier d'architecte au sein desquels la part des femmes a plus que doublé en vingt ans. Le mouvement de féminisation observé ne s'interprète cependant pas de la même façon dans l'un et l'autre cas, en raison de l'évolution très contrastée de la population de ces deux familles de métiers : dans le cas du métier d'architecte, où les effectifs ont fortement augmenté en vingt ans (+ $45 \%)$, le nombre et la part relative des femmes augmentent en parallèle. Dans le cas des métiers d'art, au contraire, les effectifs globaux se sont réduits au cours de la période $(-24 \%)$ : le nombre de femmes exerçant ces métiers demeure à peu près stable dans le temps, mais leur part relative au sein du groupe professionnel augmente en revanche considérablement car les hommes sont à la fois moins nombreux à entrer dans ces métiers et plus nombreux à les quitter avant l'âge de la retraite.

Les femmes, massivement présentes dans les activités de traduction et d'interprétariat en 1991, le demeurent vingt ans plus tard et ce, de façon plus marquée 
(respectivement $68 \%$ et $73 \%$ de femmes). À cette seule exception près, la mixité des actifs des différentes professions a donc résolument progressé. Au regard du caractère très fortement masculin de cette population professionnelle vingt ans auparavant (61\% d'hommes en 1991), ce processus est donc, pour l'essentiel, un processus de féminisation.

\section{L'emploi féminin a largement épousé les spécificités de l'emploi propres aux professions culturelles}

Dans quel cadre formel les femmes exercent-elles leur emploi dans ces professions? Sur ce plan, observe-t-on des différences significatives avec les hommes, et sont-elles de même nature que celles observées dans l'ensemble de la population active?

Le développement continu de l'emploi féminin dans l'ensemble de la population active s'est effectué simultanément à deux évolutions structurelles majeures de l'emploi : le développement du salariat et celui des emplois tertiaires. L'emploi féminin, considéré globalement, est donc prioritairement aujourd'hui un emploi salarié (92\% des femmes ont un statut salarié en 2013, contre $85 \%$ des hommes), surreprésenté dans le secteur tertiaire (où $55 \%$ des actifs en 2013 sont des femmes). En outre, depuis les années 1980, l'emploi féminin est devenu, plus souvent que l'emploi masculin, un emploi à temps partiel - ce qui n'était pas du tout le cas dans la période de forte croissance de l'emploi féminin, « exception française » parmi tous les pays d'Europe 9 .

En accédant plus largement que par le passé à l'exercice des différents métiers artistiques et culturels, les femmes y occupent-elles des emplois présentant ces mêmes caractéristiques générales?

\section{Les femmes sont plus souvent salariées que les hommes, mais elles ont investi certaines professions culturelles par le non-salariat}

Dans les professions culturelles comme dans l'ensemble de la population active, les femmes sont, en moyenne, plus souvent salariées que les hommes. Dressé en 1991, le constat vaut toujours vingt ans plus tard (graphique 3). Pour autant, la féminisation du salariat a reculé dans le champ culturel au cours de la période (l'écart entre la part des hommes et des femmes salariés passe de 15 points à 3 points entre 1991 et 2013), alors qu'elle s'est amplifiée au contraire dans l'ensemble de la population active (de 5 points à 7 points). Cette évolution propre au champ culturel tient à un double mouvement: le recul de la part des femmes salariées au cours de ces vingt années ( 6 points) et la progression inverse du salariat chez les hommes (+ 6 points).

Comment interpréter cette évolution doublement contrastée, par rapport à l'emploi artistique et culturel masculin et par rapport à l'emploi féminin en général ? Par une caractéristique propre au champ concerné : le non-salariat y est notablement plus présent que dans l'ensemble de l'emploi en France, représentant au moins un tiers des actifs des professions culturelles, de façon relativement stable depuis vingt ans. Dans l'ensemble de la population active, au contraire, il recule continûment : il concernait $15 \%$ seulement de l'emploi en 1991 et $11 \%$ en 2013 (tableau 1). Plus précisément encore, le non-salariat demeure la forme d'emploi dominante dans plusieurs métiers du champ artistique et culturel : au moins deux tiers des artistes 


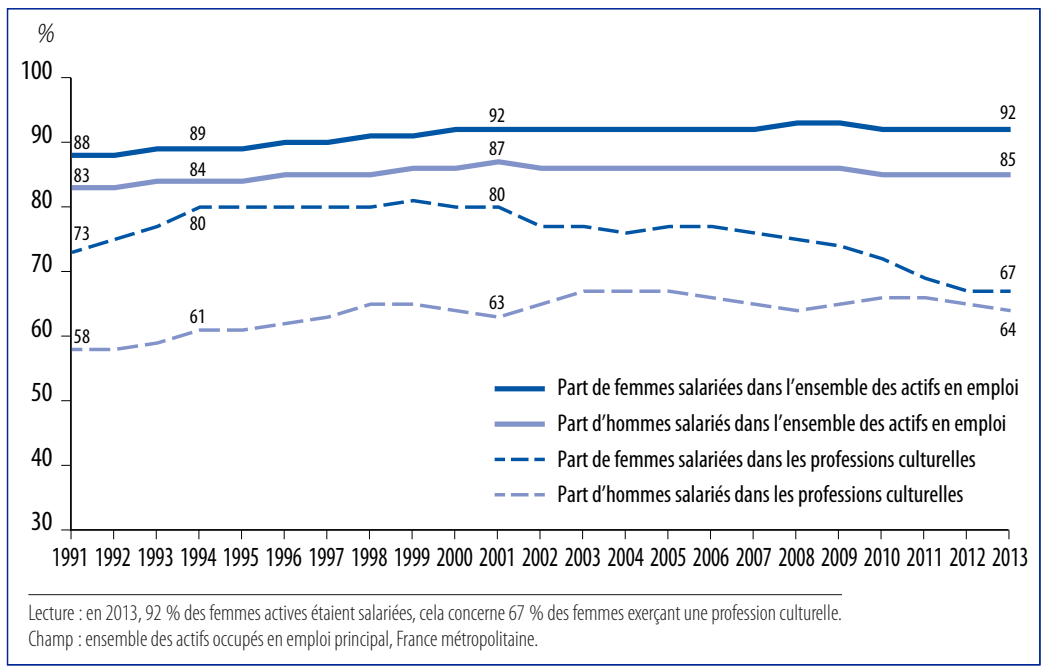

Source : Enquêtes Emploi, Insee/DEPS, Ministère de la Culture et de la Communication, 2016

plasticiens, des photographes, des auteurs littéraires et traducteurs, et une majorité des architectes et des professionnels des métiers d'art exercent leur métier sous statut indépendant. Le mouvement important de féminisation n'a pas altéré cette spécificité. Autrement dit, il s'est opéré dans les cadres statutaires habituels de l'emploi propre à chaque métier : plutôt dans le salariat, là où cette forme d'emploi était dominante (métiers du spectacle, des arts graphiques, du journalisme, de la conservation, professeurs d'art) et plutôt dans le non-salariat quand celui-ci était au contraire la forme d'emploi la plus répandue. Dans le métier de journaliste, par exemple, où le salariat domine (il concerne au moins $85 \%$ des effectifs tout au long de la période), la croissance de l'emploi féminin (+ 7 points) s'est effectuée principalement dans le cadre du salariat: la part des femmes salariées y dépasse en effet $80 \%$ de façon constante entre 1991 et 2013 (tableau 1).

Dans le métier d'artiste plasticien, s'exerçant au contraire très massivement dans le cadre du non-salariat, qui concerne plus des trois quarts des actifs, le mouvement de féminisation s'est opéré précisément dans ce cadre: la part des femmes non salariées est toujours supérieure à $80 \%$ au cours de la période, et elle dépasse même celle des hommes non salariés en 2013 (respectivement $85 \%$ et $81 \%$ ).

Cette observation générale connaît cependant deux exceptions. Dans les métiers d'art, où salariat et non-salariat représentaient des parts sensiblement équivalentes de l'emploi en 1991, la féminisation est allée de pair avec un développement marqué du non-salariat : on comptait $43 \%$ de non-salariés dans ces métiers en 1991 et $54 \%$ vingt ans plus tard, mais cela concerne respectivement $49 \%$ et $56 \%$ des femmes.

C'est l'inverse dans le cas de la profession d'architecte - traditionnellement très masculine et non salariée : le mouvement continu d'ouverture aux femmes y a épousé parfaitement la montée en puissance du salariat. Dans ce métier, les salariés représentent $38 \%$ des effectifs en 2013 (contre $35 \%$ vingt ans plus tôt), mais $54 \%$ des femmes. Au cours de la même période en revanche, la part du salariat parmi les hommes architectes est demeurée constante (autour de $30 \%$ des effectifs). 
Tableau 1 - Évolution de la part des actifs exerçant leur activité sous statut non salarié, 1991-2013

\begin{tabular}{|c|c|c|c|c|c|c|}
\hline \multirow[b]{3}{*}{ Professions culturelles } & \multirow{2}{*}{\multicolumn{2}{|c|}{$\begin{array}{c}\text { Ensemble } \\
19912013\end{array}$}} & \multirow{2}{*}{\multicolumn{2}{|c|}{$\begin{array}{c}\text { Femmes } \\
19912013\end{array}$}} & \multirow{2}{*}{\multicolumn{2}{|c|}{$\begin{array}{r}\text { Hommes } \\
19912013\end{array}$}} \\
\hline & & & & & & \\
\hline & 36 & 35 & 27 & 33 & 42 & 36 \\
\hline \multicolumn{7}{|l|}{ Professions des arts visuels et métiers d'art } \\
\hline Artistes des arts plastiques & 94 & 83 & 87 & 85 & 97 & 81 \\
\hline Photographes & 54 & 77 & 29 & 77 & 58 & 78 \\
\hline Professions des arts graphiques, de la mode et de la décoration & 29 & 40 & 25 & 38 & 33 & 42 \\
\hline Métiers d'art & 43 & 54 & 49 & 56 & 42 & 53 \\
\hline \multicolumn{7}{|l|}{ Professions des spectacles } \\
\hline Artistes des spectacles & 29 & 21 & 27 & 18 & 30 & 22 \\
\hline \multicolumn{7}{|l|}{ Cadres artistiques, de programmation } \\
\hline et de production des spectacles & 53 & 25 & 59 & 24 & 50 & 25 \\
\hline Technicien.ne.s des spectacles & 1 & 6 & 3 & 6 & 0 & 6 \\
\hline \multicolumn{7}{|l|}{ Professions littéraires } \\
\hline Journalistes et cadres de l'édition & 15 & 14 & 9 & 16 & 19 & 12 \\
\hline Auteurs littéraires & 81 & 72 & 68 & 78 & 92 & 69 \\
\hline Traducteur.rice.s et interprètes & 40 & 66 & 36 & 67 & 49 & 64 \\
\hline Architectes & 65 & 62 & 39 & 46 & 70 & 69 \\
\hline \multicolumn{7}{|l|}{$\begin{array}{l}\text { Cadres et technicien.ne.s de l'archivage, de la conservation } \\
\text { et de la documentation* }\end{array}$} \\
\hline Professeurs d'art & 26 & 21 & 31 & 21 & 16 & 23 \\
\hline Ensemble des actifs en emploi & 15 & 11 & 12 & 8 & 17 & 15 \\
\hline \multicolumn{7}{|c|}{$\begin{array}{l}\text { * Les professions de l'archivage, de la conservation et de la documentation s'exercent exclusivement de façon salariée. } \\
\text { Lecture: en 1991, } 36 \% \text { des actifs exerçant une profession culturelle étaient non salariés, ils sont } 35 \% \text { en 2013. En 1991, les femmes qui exercent un } \\
\text { profession culturelle étaient } 27 \% \text { à être non salariées, elles sont } 33 \% \text { en } 2013 \text {. En 1991, les hommes qui exercent une profession culturelle étaient } 42 \% \text { àtr } \\
\text { non salariés, ils sont } 36 \% \text { en } 2013 \text {. } \\
\text { Champ : ensemble des actifs occupés en emploi principal, France métropolitaine. }\end{array}$} \\
\hline
\end{tabular}

Source : Enquêtes Emploi, Insee/DEPS, Ministère de la Culture et de la Communication, 2016

\section{Lorsqu'elles sont salariées, les femmes sont autant concernées que les hommes par les contrats à durée limitée, mais elles le sont davantage par le temps partiel}

Dans les professions culturelles comme dans l'ensemble des professions, la part des contrats à durée limitée ( $C D D$, vacations, emplois aidés...) a doublé au sein du salariat au cours de la période (graphique 4). Ce mouvement a touché les hommes et les femmes dans des proportions comparables.

Ce qui fonde ici la spécificité du champ culturel, ce n'est pas une éventuelle différence entre hommes et femmes mais plutôt la part beaucoup plus importante qu'y occupent, globalement, les contrats de courte durée : plus de deux fois plus que dans l'ensemble de la population salariée (15\% contre $8 \%$ en 1991, $31 \%$ contre $14 \%$ en 2013). Les femmes salariées qui exercent une profession culturelle sont donc deux fois plus nombreuses que les femmes salariées en général à travailler dans le cadre de contrats courts (en 2013, par exemple : $29 \%$ contre $15 \%$ ), mais leurs homologues masculins également (en 2013, $32 \%$ des hommes salariés dans le champ culturel travaillent sous contrat à durée limitée contre $13 \%$ des hommes salariés en moyenne) (graphique 4). 


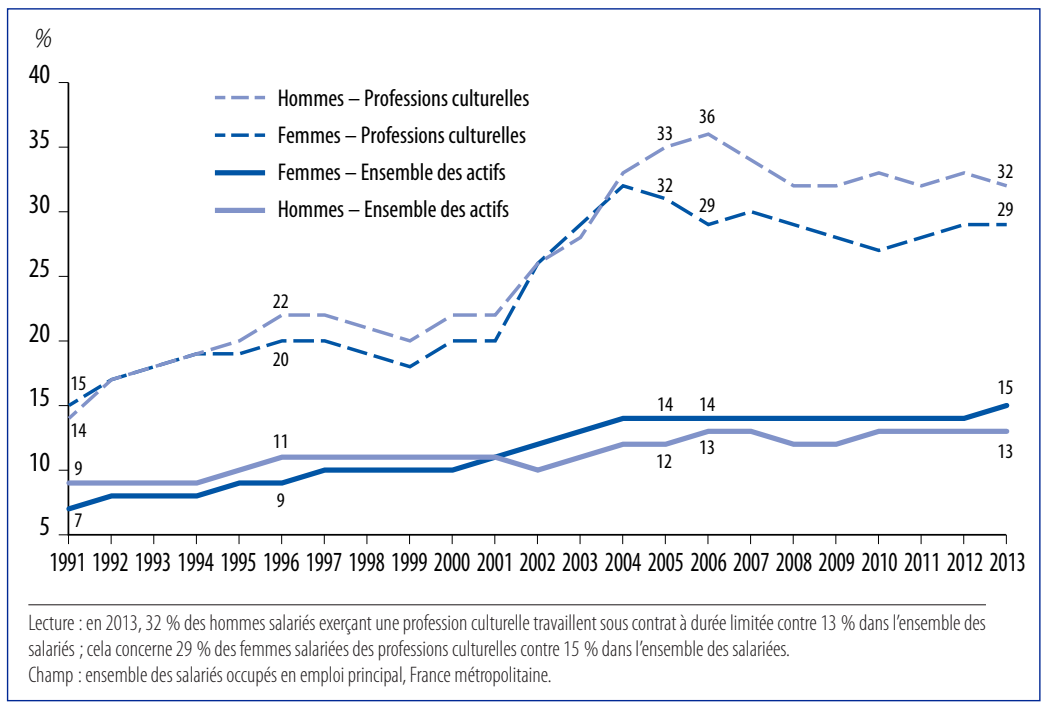

Source: Enquêtes Emploi, Insee/DEPS, Ministère de la Culture et de la Communication, 2016

II n'en va pas de même, en revanche, pour le salariat à temps partiel. Lui aussi plus répandu dans les professions culturelles que dans la population salariée dans son ensemble et en développement au cours de la période, le temps partiel est en effet plus présent chez les femmes que chez les hommes (graphique 5).

\section{Graphique 5 - Évolution de la part des femmes et des hommes salariés à temps partiel, 1991-2013}

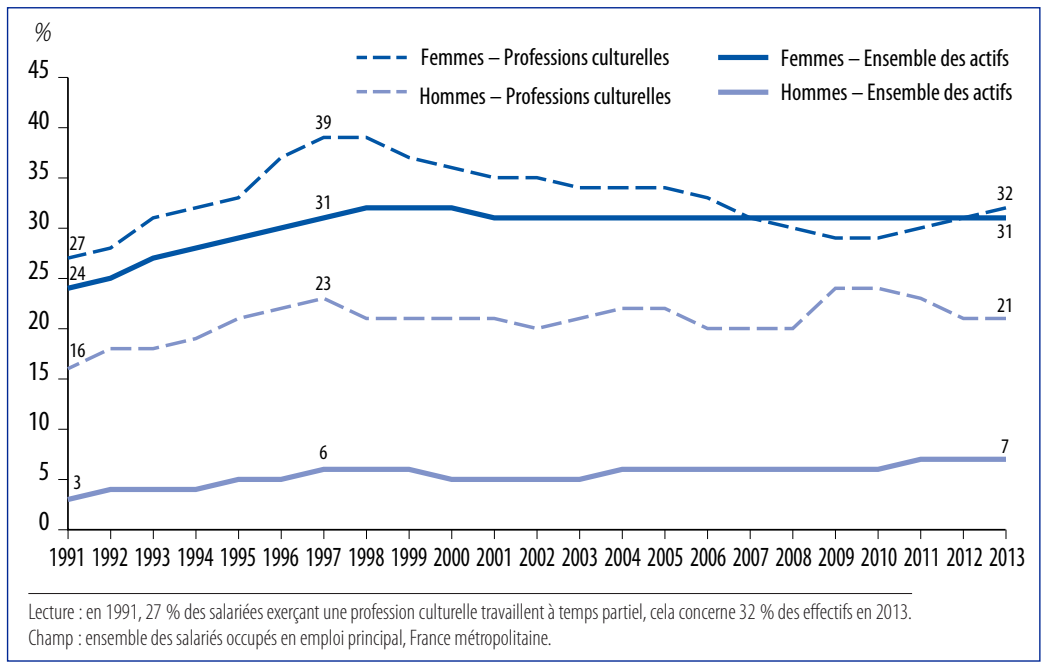


En 2013, cela concerne par exemple $32 \%$ des journalistes salariées (contre $14 \%$ des hommes), ou encore $20 \%$ des salariées des professions des arts graphiques, de la mode et de la décoration (contre $5 \%$ des hommes salariés dans ces métiers).

Ainsi, si la part des salariées à temps partiel dans l'ensemble du champ culturel a eu tendance à s'accroître depuis le début des années 1990 (de 27 \% à 32 \% entre 1991 et 2013), l'observation métier par métier ne fait pas apparaître une montée du temps partiel systématique et de grande ampleur.

À l'exception des professions de l'archivage, de la conservation et de la documentation, très massivement féminines, où le temps partiel est cinq fois plus répandu chez les femmes, toutes les professions concernées par le développement du temps partiel sont des métiers où le temps partiel est devenu dominant pour l'ensemble des salariés, hommes et femmes confondus (tableau 2). Les professeurs d'art font figure de cas emblématique sur ce point: en vingt ans, le salariat à temps partiel passe de $39 \%$ à $60 \%$, concernant une proportion comparable de femmes et d'hommes (respectivement $61 \%$ et $59 \%$ en 2013). Le cas des métiers artistiques du spectacle - autre exemple de groupe professionnel fortement marqué par le temps partiel, du fait du développement du régime de l'intermittence et de l'hyperflexibilité

\section{Tableau 2 - Caractéristiques de l'emploi salarié des professions culturelles} selon le sexe, 1991-2013

\begin{tabular}{|c|c|c|c|c|c|c|c|c|c|c|c|c|}
\hline & \multicolumn{6}{|c|}{ Salariés en contrats à durée limitée } & \multicolumn{6}{|c|}{ Salariés à temps partiel } \\
\hline & & \multicolumn{2}{|c|}{ Femmes } & \multicolumn{2}{|c|}{ Hommes } & \multicolumn{2}{|c|}{ Ensemble } & \multicolumn{2}{|c|}{ Femmes } & \multicolumn{2}{|c|}{ Hommes } \\
\hline & \multicolumn{2}{|c|}{$\overline{19912013}$} & \multicolumn{2}{|c|}{$\overline{19912013}$} & \multicolumn{2}{|c|}{$\overline{19912013}$} & \multicolumn{2}{|c|}{$\overline{19912013}$} & \multicolumn{2}{|c|}{$\overline{19912013}$} & \multicolumn{2}{|c|}{19912013} \\
\hline Professions culturelles & 15 & 31 & 14 & 29 & 15 & 32 & 21 & 26 & 27 & 32 & 16 & 21 \\
\hline \multicolumn{7}{|c|}{ Professions des arts visuels et métiers d'art } & & & \multirow[b]{2}{*}{1} & \\
\hline Artistes des arts plastiques* $^{*}$ & \multirow{2}{*}{$\begin{array}{l}1 \\
1\end{array}$} & & \multirow{3}{*}{ I } & & & & \multirow{2}{*}{1} & \multirow{2}{*}{$\begin{array}{l}1 \\
1\end{array}$} & \multirow{2}{*}{ I } & & & \\
\hline Photographes* & & & & & & & & & & & & l \\
\hline Professions des arts graphiques, & & & & & & & & & & & & \\
\hline de la mode et de la décoration & 9 & 20 & 10 & 21 & 8 & 18 & 9 & 13 & 12 & 20 & 5 & 5 \\
\hline Métiers d'art & 3 & 9 & 10 & 6 & 1 & 11 & 5 & 8 & 5 & 11 & 5 & 6 \\
\hline Professions des spectacles & & & & & & & & & & & & \\
\hline Artistes des spectacles & 41 & 70 & 51 & 72 & 36 & 69 & 45 & 47 & 40 & 58 & 47 & 42 \\
\hline Cadres artistiques, de programmati & tion & & & & & & & & & & & \\
\hline et de production des spectacles & 539 & 31 & 45 & 39 & 38 & 27 & 18 & 15 & 19 & 17 & 17 & 15 \\
\hline Technicien.ne.s des spectacles & 31 & 47 & 41 & 44 & 28 & 48 & 22 & 22 & 34 & 23 & 18 & 22 \\
\hline Professions littéraires & & & & & & & & & & & & \\
\hline s de l'édition & 8 & 19 & 12 & 21 & 5 & 17 & 12 & 22 & 19 & 32 & 7 & 14 \\
\hline Auteurs littéraire & l & l & l & l & l & l & l & l & l & l & l & l \\
\hline Traducteur.rice.s et interprètes* & l & l & l & I & l & l & I & l & l & l & l & l \\
\hline Architectes & 10 & 10 & 29 & 11 & 3 & 10 & 8 & 13 & 10 & 17 & 7 & 11 \\
\hline Cadres et $t$ & chivas & ge, de I & a conse & ervation & & & & & & & & \\
\hline et de la documentation & 6 & 8 & 8 & 11 & 1 & 2 & 28 & 15 & 32 & 20 & 12 & 4 \\
\hline Professeurs d'art & 5 & 26 & 6 & 25 & 4 & 27 & 39 & 60 & 50 & 61 & 23 & 59 \\
\hline Ensemble des salariés en emploi & i 8 & 14 & 7 & 15 & 9 & 13 & 12 & 19 & 24 & 31 & 3 & 7 \\
\hline
\end{tabular}




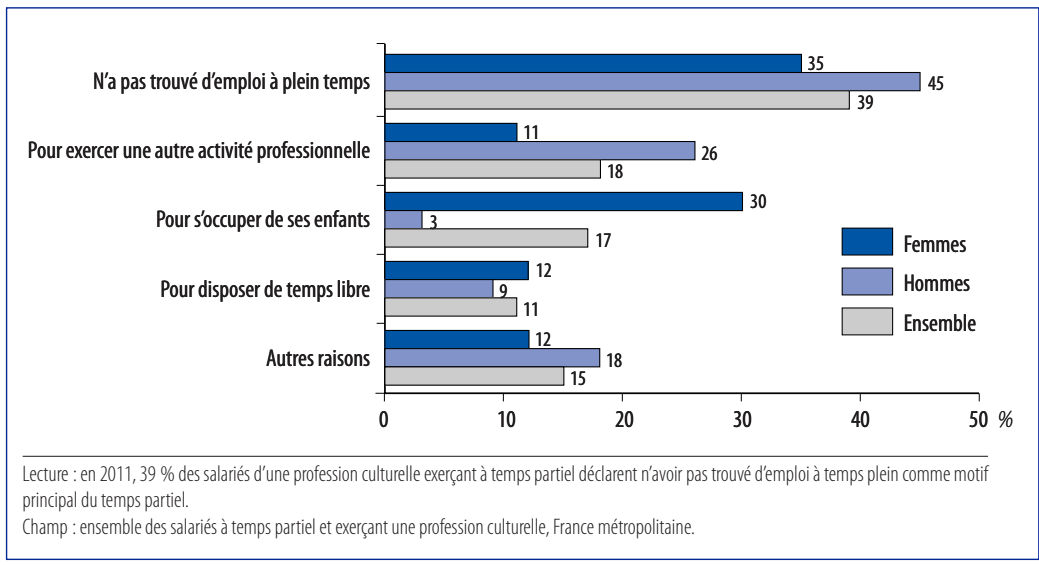

Source : Enquêtes Emploi, Insee/DEPS, Ministère de la Culture et de la Communication, 2016

salariale ${ }^{10}$ - s'inscrit un peu en marge de ce constat : le temps partiel continue globalement à s'y développer (passant de 45\% en 1991 à 47\% en 2013), mais cette tendance est due exclusivement à sa progression au sein du salariat féminin (+ 18 points entre les deux dates).

Interrogés en 2011 sur la raison principale qui motive l'exercice du travail à temps partiel, les salariés des professions culturelles répondent le plus souvent qu'ils n'ont pas trouvé d'emploi à temps plein : les femmes comme les hommes citent ce motif au premier rang, avec toutefois une part plus modeste de femmes ${ }^{11}$ (35\% contre $45 \%$ des hommes salariés à temps partiel) (graphique 6). La seconde raison la plus fréquemment évoquée distingue en revanche très fortement les salariés des deux sexes: du côté des femmes, il s'agit du choix de s'occuper de leurs enfants (30 \% contre seulement $3 \%$ des hommes) et du côté des hommes, des contraintes liées à l'exercice d'une seconde activité professionnelle ( $26 \%$ des hommes mais seulement $11 \%$ des femmes).

\section{Des modalités d'organisation du travail atypiques, mais dans une proportion plutôt moindre pour les femmes que pour les hommes}

Dans les professions culturelles, le travail s'inscrit souvent en dehors des cadres temporels les plus répandus parmi l'ensemble des actifs : le travail du soir, de nuit et du dimanche y est ainsi notablement plus fréquent qu'en moyenne, ainsi que la variabilité des horaires d'une semaine sur l'autre (tableau 3). Cette plus grande plasticité, il est vrai, tend aujourd'hui à se développer dans d'autres professions ne relevant pas de la sphère culturelle ${ }^{12}$. Mais dans ce champ précis, elle tient fortement aux conditions d'exercice intrinsèques de certains métiers ${ }^{13}$ (par exemple : jours et horaires des spec-

10. Pierre-Michel MENGER, les Intermittents du spectacle: sociologie du travail flexible, Paris, Éditions de I'EHESS, 2011 ; Marie GouYon et Frédérique PATUREAU, Tendances de l'emploi dans le spectacle, Paris, Ministère de la Culture et de la Communication, DEPS, coll. "Culture chiffres », n² 2014-2, 2014.

11. La question n'était pas posée en 1991 et n'est pas exploitable en 2013 suite à un changement des modalités de réponse (voir Source et méthode, p. 19).

12. Voir Jean-David FERMANIAN et Pierre BOISARD, "Les rythmes de travail hors norme ", Économie et Statistique, $\mathrm{n}^{\circ} 321-322,1999$.

13. Jérémy SINIGAGLIA et Sabrina SINIGAGLIA-AmADIO, les Temporalités du travail artistique, Paris, Ministère de la Culture et de la Communication, coll. «Questions de culture », à paraître, 2017. 


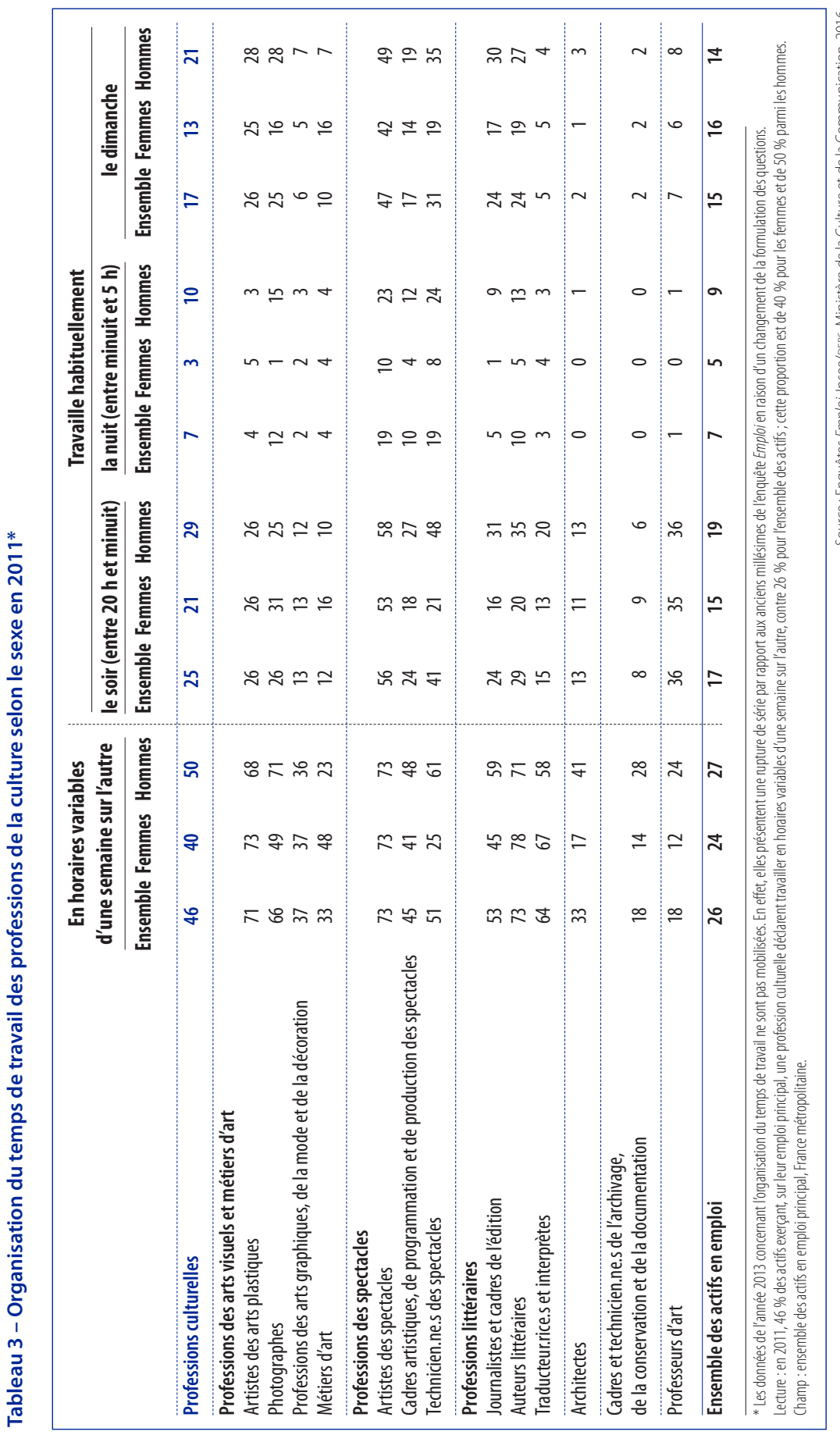


tacles, des tournages pour les professionnels des spectacles; temps de conception et/ou de préparation des œuvres et réalisations pour les plasticiens; pression de l'actualité pour les journalistes, etc.). Elle tient aussi à la surreprésentation du nonsalariat qui, de façon générale, s'exerce dans une plus grande plasticité horaire et hebdomadaire ${ }^{14}$ et qui est parfois soumis à des surcharges temporaires d'activité (liées à l'irrégularité des commandes, par exemple: cas des architectes, des photographes...).

De ce fait, les femmes des professions culturelles sont plus concernées que la moyenne des femmes actives par les horaires variables d'une semaine sur l'autre (40\% contre $24 \%$ parmi l'ensemble des actives) ou par le travail en soirée (respectivement $21 \%$ et $15 \%$ ). Mais elles le sont cependant toujours moins que les hommes exerçant les mêmes métiers : un homme sur deux exerçant une profession culturelle est soumis à des horaires variables (tableau 3). II est très rare en revanche qu'elles travaillent habituellement de nuit (3\% contre $10 \%$ des hommes), plus rare même que dans l'ensemble de la population active féminine où le travail nocturne est courant dans certains métiers fortement féminins (comme ceux de la santé, par exemple).

L'emploi féminin dans les métiers culturels présente donc certains traits communs avec l'emploi féminin dans son ensemble : les femmes y sont plus souvent salariées, plus souvent à temps partiel, notamment. Mais deux points, surtout, ressortent de l'analyse. Le fait, tout d'abord, qu'en investissant plus largement ce champ professionnel, les femmes ont épousé les caractéristiques d'emploi les plus répandues au sein de chaque métier : dans l'art et la culture, elles sont donc, à peu près autant que les hommes, non salariées, salariées sur contrats à durée déterminée, en horaires variables et décalés, etc. Le fait, ensuite, que de très grands contrastes s'observent d'une profession à l'autre. À ce titre, l'observation des métiers au sein desquels le mouvement de féminisation a été le plus marqué permet-elle de repérer des traits communs, susceptibles d'avoir facilité cette progression?

\section{Les voies de la féminisation}

On l'a vu : en vingt ans, la croissance des actifs dans les professions culturelles a été bien plus rapide que celle de la population active dans son ensemble $(+50 \%$ contre $+16 \%)$. Mais la progression de la part des femmes n'a pas suivi mécaniquement celle des actifs des différents métiers.

\section{Les professions qui se sont les plus féminisées en vingt ans ne sont pas celles qui ont connu la plus forte croissance de leurs effectifs}

Les deux familles de métiers qui ont connu la plus forte expansion numérique - celle des techniciens des spectacles (+ $148 \%$ entre 1991 et 2013) et celle des professions des arts graphiques, de la mode et de la décoration (+ 107\%) - n'affichent qu'une très faible féminisation de leurs effectifs au cours de la période (respectivement +0 point et +4 points).

Les professions des arts graphiques, de la mode et de la décoration étaient déjà, il est vrai, quasiment paritaires vingt ans plus tôt (48\% de femmes en 1991), ce qui peut expliquer la faible amplitude du mouvement de féminisation. Mais les professions

14. Élisabeth ALGAVA et Lydie VINCK, "Les conditions de travail des non-salariés en 2005 ", Premières Synthèses, n 50-1, décembre 2009; Michel AMAR et Franck EvAIN, "Les indépendants », Insee Première, n० 1084, juin 2006; Marie Gouyon, "Les non-salariés dans les activités culturelles », Insee Références, février 2015. 
techniques des spectacles, très masculines au contraire, ne se sont quasiment pas ouvertes aux femmes qui ne représentent qu'un quart à un tiers des effectifs tout au long de la période, en dépit du doublement de la population. Pour cette dernière famille de métiers, les freins à la féminisation sont vraisemblablement multiples et cumulatifs. Le contenu très technique des tâches, la pénibilité physique, la plasticité des horaires de travail et leur irrégularité - toutes caractéristiques généralement associées à l'exercice de ces métiers - ne peuvent suffire à rendre compte de cette difficile percée des femmes, car ces mêmes caractéristiques de travail s'appliquent largement aux métiers artistiques des spectacles (comédiens, danseurs, musiciens...), traditionnellement plus féminins et qui ont connu dans le même temps un mouvement de féminisation plus marqué.

Il faut donc à l'évidence invoquer d'autres freins, plus invisibles. On pense ici aux modalités d'accès à ces métiers (formation professionnelle, processus de recrutement) dont les recherches récentes sur la féminisation ont révélé toute l'importance. Cellesci ont mis en évidence le lien entre la féminisation de certains métiers traditionnellement masculins et l'introduction d'un plus grand formalisme dans leur accès : création de formations qualifiantes et de diplômes spécialisés, énoncé de règles formelles régissant l'accès à ces formations professionnelles, définition des modalités de recrutement ${ }^{15}$. L'accès à certains métiers techniques du spectacle, au contraire, reste peu formalisé (c'est-à-dire non strictement subordonné au suivi d'une formation et à la détention d'un diplôme spécifique) et surtout, les modalités de recrutement font une large place à la cooptation par les pairs, au sein de réseaux professionnels essentiellement (voire exclusivement) masculins ${ }^{16}$. La surreprésentation masculine au sein des instances de régulation professionnelle (syndicats, coordinations, groupements) et, sans doute, l'anticipation d'une plus grande difficulté à s'insérer durablement dans ces métiers et à y progresser contribuent vraisemblablement à décourager les vocations féminines à l'exercice de ces professions ${ }^{17}$.

Les professions culturelles qui se sont les plus ouvertes aux femmes en vingt ans sont plutôt des professions qui ont connu une croissance modérée de leurs effectifs globaux (artistes plasticiens, photographes, journalistes, architectes) ou même, dans le cas des métiers d'art, une décroissance de ceux-ci. On observe, par ailleurs, que ces professions étaient aussi, en début de période, plutôt très masculines : le mouvement d'ouverture aux femmes peut donc y apparaître comme une forme de rattrapage vers la parité. Dans le cas précis de la profession d'architecte, la féminisation s'est effectuée simultanément avec le développement du salariat, on l'a vu, mais aussi avec l'apparition de nouvelles règles formelles pour l'entrée en formation ${ }^{18}$ : la part chaque année croissante des jeunes femmes dans les écoles d'architecture (+ $20 \%$ entre l'année universitaire 1990-1991 et 2011-2012 ${ }^{19}$ ) laisse présager une poursuite mécanique de la féminisation du métier.

\section{Diplôme et origine sociale favorisée : la règle de la sursocialisation des femmes}

L'une des spécificités les plus marquées et les plus constantes des professions culturelles est qu'elles sont exercées par des personnes dotées d'un haut niveau de diplôme - et ce, même lorsque l'accès à ces métiers n'est pas formellement conditionné

15. Voir Nathalie LAPEYRE, les Professions face aux enjeux de la féminisation, Paris, Octarès, 2006.

16. Marie BusCATTO, "La féminisation du travail artistique à l'aune des réseaux sociaux », Sociologie de l'art, $\mathrm{n}^{\circ}$ 2014/2-3, 2014, p. 129-152.

17. Vincent CARDON, Janine RANNOU et lonela RoHARIK, les Inégalités hommes/femmes dans l'audiovisuel au travers des dynamiques de parcours, Observatoire des métiers de l'audiovisuel, avril 2015.

18. Nathalie LAPEYRE, les Professions face aux enjeux de la féminisation, op. cit.

19. Source : Ministère de l'Enseignement supérieur et de la Recherche, DGESIP-DGRI SIES. 
par la détention d'un diplôme spécifique, ce qui est le plus souvent le cas des métiers de création (tableau 4). Cette caractéristique désormais bien connue est liée au contenu des métiers et au niveau de qualification et d'autonomie requis pour les exercer, mais aussi, très largement, à l'origine familiale socialement favorisée des personnes ${ }^{20}$.

En 1991, plus d'un professionnel exerçant un métier culturel sur quatre détenait un diplôme au moins égal à bac + 3 (contre $9 \%$ seulement des actifs en moyenne), et cette part s'est élevée continûment pour atteindre 45 \% en 2013 (contre 21 \% de l'ensemble des actifs à cette date).

Quelle position les femmes occupent-elles dans ce mouvement?

La question revêt une certaine importance, si l'on se souvient qu'il s'agit d'une tendance de fond qui a touché l'ensemble de la population active mais tout particulièrement les femmes, premières bénéficiaires des progrès de la massification scolaire et universitaire et de l'allongement de la durée d'études.

\section{Tableau 4 - Part des diplômés de l'enseignement supérieur* parmi les professionnels} de la culture selon le sexe, 1991-2013

\begin{tabular}{|c|c|c|c|c|c|c|}
\hline \multirow[b]{3}{*}{ Professions culturelles } & \multirow{2}{*}{\multicolumn{2}{|c|}{$\begin{array}{l}\text { Ensemble } \\
19912013\end{array}$}} & \multirow{2}{*}{\multicolumn{2}{|c|}{$\begin{array}{c}\text { Femmes } \\
19912013\end{array}$}} & \multirow{2}{*}{\multicolumn{2}{|c|}{$\begin{array}{r}\text { Hommes } \\
19912013\end{array}$}} \\
\hline & & & & & & \\
\hline & 26 & 45 & 31 & 52 & 23 & 40 \\
\hline \multicolumn{7}{|l|}{ Professions des arts visuels et métiers d'art } \\
\hline Artistes des arts plastiques & 24 & 41 & 28 & 55 & 21 & 31 \\
\hline Photographes & 6 & 33 & 13 & 50 & 5 & 26 \\
\hline Professions des arts graphiques, de la mode et de la décoration & 8 & 36 & 6 & 35 & 10 & 37 \\
\hline Métiers d'art & 2 & 18 & 7 & 33 & 1 & 7 \\
\hline \multicolumn{7}{|l|}{ Professions des spectacles } \\
\hline Artistes des spectacles & 12 & 41 & 14 & 47 & 11 & 38 \\
\hline \multicolumn{7}{|l|}{ Cadres artistiques, de programmation } \\
\hline et de production des spectacles & 15 & 41 & 19 & 52 & 13 & 36 \\
\hline Technicien.ne.s des spectacles & 7 & 21 & 16 & 33 & 5 & 17 \\
\hline \multicolumn{7}{|l|}{ Professions littéraires } \\
\hline Journalistes et cadres de l'édition & 44 & 63 & 46 & 68 & 42 & 60 \\
\hline Auteurs littéraires & 42 & 59 & 53 & 62 & 34 & 57 \\
\hline Traducteur.rice.s et interprètes & 59 & 72 & 63 & 78 & 51 & 56 \\
\hline Architectes & 70 & 86 & 76 & 94 & 68 & 82 \\
\hline $\begin{array}{l}\text { Cadres et technicien.ne.s de l'archivage, de la conservation } \\
\text { et de la documentation }\end{array}$ & 47 & 68 & 48 & 69 & 40 & 67 \\
\hline Professeurs d'art & 30 & 42 & 31 & 50 & 28 & 31 \\
\hline Ensemble des actifs en emploi & 9 & 21 & 8 & 22 & 10 & 20 \\
\hline \multicolumn{7}{|c|}{$\begin{array}{l}\text { *Titulaires d'un diplôme de niveau égal ou supérieur à bac + } 3 \text {. } \\
\text { Lecture : en 1991,23\% des hommes exerçant une profession culturelle étaient titulaires d'un diplôme de niveau égal ou supérieur à bac + } 3 \text {; ils sont } 40 \% \text { \% } \\
\text { vingt ans plus tard. }\end{array}$} \\
\hline
\end{tabular}

Source: Enquêtes Emploi, Insee/DEPS, Ministère de la Culture et de la Communication, 2016

20. Marie Gouyon et Frédérique PaturEau, Vingt ans d'évolution de l'emploi dans les professions culturelles, op. cit. 
Dans les professions culturelles, la règle de la surqualification des femmes en termes de niveau de diplôme est amplement vérifiée. Elle s'impose même de façon plus marquée que dans l'ensemble de la population active. Ainsi, en 1991, $26 \%$ des actifs des professions culturelles étaient titulaires d'un diplôme au moins égal à bac +3 , mais cette part s'élevait déjà à $31 \%$ chez les femmes. Vingt ans plus tard, plus d'une active sur deux (52\%) des professions culturelles détient ce niveau de diplôme contre $40 \%$ de leurs homologues masculins.

Tous les métiers sans exception sont concernés, y compris ceux qui étaient déjà très fortement diplômés en 1991 (journalistes, auteurs littéraires, traducteurs, architectes...). II n'y a donc pas ici un simple effet de rattrapage propre à certains métiers exercés par des actifs moins diplômés au cours de la période précédente.

Ainsi, au sein de la population des auteurs littéraires, fortement diplômée (42\% des effectifs étaient titulaires d'un diplôme de niveau bac + 3 et plus en 1991, 59 \% le sont vingt ans plus tard), la part des femmes diplômées de l'enseignement supérieur reste supérieure à celle de leurs homologues masculins : en 2013, $62 \%$ des femmes contre $57 \%$ des hommes sont titulaires d'un diplôme de niveau égal ou supérieur à bac +3 . Il en va de même dans les professions de traduction et d'interprétariat, certes plus féminines, où la part de femmes titulaires d'un diplôme de niveau égal ou supérieur à bac +3 est supérieure de plus de vingt points à celle des hommes exerçant les mêmes professions (78\% contre $56 \%$ ).

On le voit, cette caractéristique générale de l'emploi féminin dans les professions culturelles s'est encore accrue au cours des vingt dernières années, d'autant plus fortement, semble-t-il, qu'il s'agissait d'un métier traditionnellement exercé par des hommes.

Chez les techniciens des spectacles, par exemple, où la part des femmes est modeste et le demeure au cours de la période (entre $25 \%$ et $30 \%$ ), l'écart de niveau de diplôme en faveur des femmes s'accroît fortement : on compte $7 \%$ de diplômés de niveau bac +3 et plus dans ces métiers en 1991, mais $16 \%$ des femmes et $5 \%$ seulement des hommes ; $21 \%$ en 2013, mais $33 \%$ des femmes et moitié moins d'hommes (17\%).

Pour la profession d'architecte, où la part de diplômés du supérieur (de niveau bac +3 et plus) passe de $70 \%$ à $86 \%$ en vingt ans, l'avantage des femmes en termes de niveau de diplôme passe de 8 points à 11 points au cours de la période, et dans les métiers d'art, de 5 points à 26 points. Pour ces deux derniers métiers, très masculins en début de période, l'avantage particulièrement marqué des femmes en termes de diplôme peut donc s'interpréter comme un levier important du mouvement de féminisation observé au cours des vingt dernières années - et comme un droit d'entrée plus élevé pour les femmes, dans leur accès à des métiers traditionnellement masculins ${ }^{21}$.

Corrélée à la variable du niveau de diplôme, celle de l'origine sociale confirme et renforce le constat d'une sursocialisation des femmes dans les professions culturelles. Dans ce champ en effet, l'origine sociale des actifs des deux sexes fait nettement apparaître une surreprésentation des enfants de cadres : deux fois et demie plus que dans l'ensemble de la population active en 1991, deux fois plus en 2013 (tableau 5).

21. Ce surclassement des femmes en termes de niveau de diplôme est attesté par de nombreuses recherches concernant des métiers du champ artistique (Delphine NAUDIER, «Les modes d'accès des femmes écrivains au champ littéraire contemporain », dans Gérard MAUGER (sous la dir. de), Droits d'entrée. Modalités et conditions d'accès aux univers artistiques, Paris, Éditions de la MSH, 2007; Hyacinthe RAVET, "Professionnalisation féminine et féminisation d'une profession. Les artistes interprètes de la musique ", Travail, Genre et Sociétés, n 9, 2003) et plus généralement, des métiers fortement masculins, toutes professions confondues (voir notamment Catherine MARRY, « Une féminisation de la profession d'ingénieur en France et en Allemagne ", dans Paul BOUfFARTIGUE [sous la dir. de]), Cadres : Ia grande rupture, Paris, La Découverte, 2001. Voir aussi Marlaine CACOUAULt-BITAUD et Christiane FourniER, "Le diplôme contribue-t-il à réduire les différences entre hommes et femmes sur le marché du travail ? ", dans Nicole MoscoNI (sous la dir. de), Égalité des sexes en éducation et formation, Paris, PUF, coll. «Éducation et formation », 1998. 


\begin{tabular}{|c|c|c|c|c|c|c|}
\hline \multirow[b]{3}{*}{ Professions culturelles } & \multicolumn{2}{|c|}{ Ensemble } & \multirow{2}{*}{\multicolumn{2}{|c|}{$\begin{array}{c}\text { Femmes } \\
19912013\end{array}$}} & \multirow{2}{*}{\multicolumn{2}{|c|}{$\begin{array}{c}\text { Hommes } \\
19912013\end{array}$}} \\
\hline & \multicolumn{2}{|c|}{19912013} & & & & \\
\hline & 45 & 52 & 49 & 53 & 42 & 51 \\
\hline Professions des arts visuels et métiers d'art & & & & & & \\
\hline Artistes des arts plastiques & 40 & 59 & 52 & 63 & 33 & 56 \\
\hline Photographes & 44 & 49 & 30 & 63 & 46 & 43 \\
\hline Professions des arts graphiques, de la mode et de la décoration & 44 & 51 & 49 & 50 & 38 & 52 \\
\hline Métiers d'art & 21 & 32 & 36 & 39 & 18 & 27 \\
\hline Professions des spectacles & & & & & & \\
\hline Artistes des spectacles & 54 & 59 & 59 & 56 & 51 & 60 \\
\hline Cadres artistiques, de programmation & & & & & & \\
\hline et de production des spectacles & 39 & 47 & 36 & 50 & 40 & 45 \\
\hline Technicien.ne.s des spectacles & 39 & 46 & 57 & 42 & 33 & 47 \\
\hline Professions littéraires & & & & & & \\
\hline Journalistes et cadres de l'édition & 58 & 58 & 55 & 57 & 60 & 58 \\
\hline Auteurs littéraires & 60 & 63 & 69 & 65 & 52 & 62 \\
\hline Traducteur.rice.s et interprètes & 53 & 55 & 58 & 61 & 41 & 37 \\
\hline Architectes & 57 & 56 & 60 & 62 & 56 & 53 \\
\hline $\begin{array}{l}\text { Cadres et technicien.ne.s de l'archivage, de la conservation } \\
\text { et de la documentation }\end{array}$ & 46 & 54 & 47 & 51 & 43 & 61 \\
\hline Professeurs d'art & 44 & 50 & 44 & 51 & 43 & 48 \\
\hline Ensemble des actifs en emploi & 19 & 27 & 19 & 27 & 18 & 26 \\
\hline $\begin{array}{l}\text { * Le père appartient (appartenait) aux catégories « cadres et professions intellectuelles } \\
\text { Lecture : en 2013, } 53 \% \text { des femmes exerçant une profession culturelle avaient un perr } \\
\text { culturelle. } \\
\text { Champ : ensemble des actifs en emploi principal, France métropolitaine. }\end{array}$ & $\begin{array}{l}\text { upérieures } \\
\text { adre, cela }\end{array}$ & $\begin{array}{l}\text { " et « pro } \\
\text { oncernait }\end{array}$ & & tiaires, & & \\
\hline
\end{tabular}

Source: Enquêtes Emploi, Insee/DEPS, Ministère de la Culture et de la Communication, 2016

Mais les femmes, ici encore, se distinguent de leurs homologues masculins par une origine sociale plus favorisée (+ 7 points en 1991, +2 points en 2013).

Cette observation générale ne concerne cependant pas toutes les professions culturelles au même titre. Elle se vérifie tout spécialement dans les métiers très masculins en début de période, qui se sont féminisés par la suite : le métier de photographe par exemple, encore peu féminisé au début des années 1990, où la part de femmes ayant un père cadre excède désormais de 20 points celle des hommes de la même origine familiale. Les métiers d'art en sont un autre exemple puisque la part d'actives ayant un père cadre excède de 12 points en 2013 celle de leurs homologues masculins.

Origine sociale et niveau de diplôme plus élevés - cumul des ressources scolaires et familiales - apparaissent donc pour les femmes, dans le champ des professions culturelles comme dans d'autres ${ }^{22}$, comme des atouts essentiels pour investir les professions traditionnellement les plus masculines.

22. Voir par exemple : Catherine MARRY, les Femmes ingénieurs. Une révolution respectueuse, Paris, Belin, coll. «Perspectives sociologiques », 2004. 


\section{Les disparités de revenus dans les professions culturelles, d'après l'enquête Revenus fiscaux et sociaux de I'Insee}

Une exploitation pionnière récente de l'enquête Revenus fiscaux et sociaux de l'Insee permet une première analyse des revenus dans les professions culturelles sous l'angle des disparités hommes/femmes.

En ce qui concerne les revenus d'activité moyens (incluant d'éventuels revenus de remplacement comme le chômage), les femmes des professions culturelles (toutes professions confondues) perçoivent des revenus inférieurs en moyenne de $19 \%$ à ceux des hommes des mêmes professions (soit 22900 euros annuels moyens pour les femmes et 28300 euros pour les hommes).

Cet écart, très variable d'une profession à l'autre, est en moyenne inférieur à celui observé dans l'ensemble de la population active ( $29 \%$ d'écart entre les actifs des deux sexes).

Si l'on considère l'ensemble des revenus du ménage (revenus d'activité + revenus du conjoint, prestations sociales, revenus fonciers, revenus financiers...), l'écart hommes/femmes s'inverse cependant: les professionnelles de la culture (plus souvent en couple avec un conjoint cadre: $73 \%$ d'entre elles) retrouvent une légère avance sur leurs collègues hommes (respectivement 28800 euros et 27500 euros annuels en moyenne).

Source: Marie Gouyon, Revenus d'activité et niveaux de vie des professionnels de la culture, Paris, Ministère de la Culture et de la Communication, DEPS, coll. « Culture chiffres », n 2015-1, 2015.

\section{Le champ des métiers artistiques et culturels dans la nomenclature PCS}

Vingt-trois métiers spécifiques du domaine des arts, des spectacles, de la culture et de l'information ont été isolés au sein des 455 professions recensées par la nomenclature des professions et catégories socioprofessionnelles (PCS). Il s'agit des métiers (ou groupes de métiers) suivants:

\section{Professions des arts visuels}

$354 \mathrm{~A}$ Artistes plasticiens

465C Photographes

465A Concepteurs et assistants techniques

des arts graphiques, de la mode et de la décoration

\section{Professions des arts visuels et métiers d'art}

Métiers d'art

214E Artisans d'art

637B Ouvriers d'art

\section{Professions des spectacles}

\section{Artistes des spectacles}

$354 \mathrm{~B}$ Artistes de la musique et du chant

$354 C$ Artistes dramatiques

354D Artistes de la danse, du cirque et des spectacles divers

\section{Techniciens des spectacles}

465B Assistants techniques

637C Ouvriers et techniciens
Cadres artistiques, de programmation et de production des spectacles

353B Directeurs, responsables de programmation et de production

353C Cadres artistiques et technico-artistiques

227A Indépendants gestionnaires de spectacles ou de services récréatifs

\section{Journalistes et cadres de l'édition}

$352 \mathrm{~A}$ Journalistes et rédacteurs en chef

353A Directeurs de journaux, administrateurs de presse, directeurs d'édition

\section{Professions littéraires}

Auteurs littéraires et traducteurs

352B Auteurs littéraires, scénaristes, dialoguistes

464B Traducteur.rice.s et interprètes

\section{Architectes}

312F Architectes libéraux

382B Architectes salariés

\section{Professions de la documentation, de l'archivage et de la conservation}

351A Bibliothécaires, archivistes, conservateurs, de la fonction publique

372F Cadres de la documentation, de l'archivage (hors fonction publique)

\section{Professeurs d'art (hors établissements scolaires)}

354G Professeurs d'art (hors établissements scolaires d'enseignement général) 


\section{Portrait sociodémographique}

En 2013, les femmes exerçant une profession culturelle sont en moyenne légèrement plus jeunes que les hommes de ces métiers (42 ans contre 43 ans) et du même âge en moyenne que l'ensemble des actives. Les plus jeunes (37 ans en moyenne) travaillent en tant que techniciennes des spectacles et les plus âgées sont artistes plasticiennes (50 ans en moyenne).

Elles sont plus diplômées que l'ensemble des femmes actives et des hommes exerçant les mêmes professions qu'elles : 52 \% sont titulaires d'un diplôme égal ou supérieur à bac + 3 contre $40 \%$ des hommes des mêmes métiers culturels et $22 \%$ des actives en général.

Plus d'une femme exerçant une profession culturelle sur deux a un père cadre (53\%, contre $51 \%$ des hommes des mêmes professions et $27 \%$ des femmes actives en général).

Caractéristique au sein des professions culturelles, elles habitent plus souvent en îlede-France (40\% contre $21 \%$ seulement de l'ensemble des femmes actives).

Le fait de vivre en couple est moins fréquent pour les femmes des professions culturelles que pour les actives en général ( $65 \%$ d'entre elles vivent en couple contre $72 \%$ de l'ensemble des actives). Six actives des professions culturelles sur dix n'ont pas d'enfants de moins de 18 ans dans leur ménage contre une active sur deux dans l'ensemble de la population.

\section{Source et méthode}

Toutes les données statistiques présentées dans cette note sont tirées d'une analyse des enquêtes Emploi (Insee) de 1990 à 2014 réalisée par le DEPS.

Conçues et menées par l'Insee, ces enquêtes interrogent un échantillon d'individus âgés de 15 ans ou plus résidant en France métropolitaine sur l'emploi, le chômage, la formation, l'origine sociale ou encore la situation d'emploi un an auparavant.

Annuelle depuis sa création en 1950, l'enquête Emploi est devenue trimestrielle en 2003.

Avant 2003, les enquêtes Emploi interrogeaient environ 70000 individus chaque année au mois de mars. Depuis 2003, la collecte s'opère en continu, sur toutes les semaines de chaque trimestre.

Un même logement est interrogé six fois (les différentes vagues étant espacées exactement d'un trimestre). Au total, l'enquête interroge désormais, chaque trimestre, 108000 individus.

La présente étude repose sur la fusion de trois enquêtes consécutives (1990 à 1992, d'une part, 2012 à 2014, d'autre part), afin de garantir la robustesse et la fiabilité des résultats sur le champ restreint que constituent les métiers artistiques et culturels, en disposant ainsi d'effectifs plus importants.

On a choisi de raisonner en moyenne annuelle: 1991 (pour la période d'enquête 1990-1992) et 2013 (pour la période d'enquête 2012-2014).

Le questionnaire de l'enquête a été rénové au $1^{\text {er }}$ trimestre 2013 sans que cela n'ait eu d'impact sur la mesure de l'emploi culturel dans ses grandes caractéristiques. Cependant, il existe une rupture de série quant aux modalités des questions sur l'organisation du travail (travail du soir, nuit et dimanche) et les raisons du temps partiel qui conduit à présenter des données en moyenne annuelle sur 2011 et non 2013. 


\section{Abstract}

\section{The Gradual Feminisation of the Cultural Professions}

Since the 1960s, one of the major transformations in the professional sphere has been the increasing proportion of women in work, and the cultural professions are no exception: the proportion of women in the cultural professions has risen from 39\% to 43\% between 1991 and 2013. Almost all the male-dominated professions of the early 1990s, for example art, architecture and photography, have since opened up to women. Nevertheless, the proportion of women remains below the nation al average, where women represent $48 \%$ of all professions; this might be considered surprising when one considers the overrepresentation of women when it comes to cultural participation.

Whilst on the increase, jobs for women have broadly embraced the employment conditions prevalent in the cultural professions, such as their greater levels of flexibility: female workers in the cultural professions are, more often than female workers overall, unsalaried or working short-term contracts. They are more likely to work part-time, with variable and irregular hours, and work evenings and weekends.

Moreover, their access to the artistic and cultural professions confirms the view that women tend to be educationally overqualified: in 2013, over $50 \%$ of female workers in these professions have completed at least 3 years of higher education, as compared with their male counterparts, about $40 \%$ - an even greater level of overqualification given that these are traditionally maledominated professions.

Directeur de la publication : Loup Wolff, chef du Département des études, de la prospection et des statistiques Responsable de la publication : Edwige Millery

\section{Retrouvez l'ensemble des publications du DEPS : http://www.culturecommunication.gouv.fr/Etudes-et-statistiques http://www.cairn.info/editeur.php?iD_EDITEUR=DEPS http://www.books.openedition.org/deps}

Le DEPS n'assurant pas de diffusion physique de ses collections de synthèse, nous vous proposons de vous informer régulièrement des parutions par message électronique.

Pour ce faire, merci de bien vouloir nous communiquer votre courriel à l'adresse contact.deps@culture.gouv.fr 